\title{
COMMUTATIVE RINGS OVER WHICH ALGEBRAS GENERATED BY IDEMPOTENTS ARE QUOTIENTS OF GROUP ALGEBRAS
}

\author{
HIDEYASU KAWAI AND NOBUHARU ONODA
}

\begin{abstract}
We study the relationship between algebras generated by idempotents over a commutative ring $R$ with identity and algebras that are quotient rings of group algebras $R G$ for torsion abelian groups $G$ without an element whose order is a zero-divisor in $R$. The main purpose is to seek conditions for $R$ to hold the equality between these two kinds of algebras.
\end{abstract}

1. Introduction. In this paper, we fix a commutative ring $R$ with identity $1_{R}$, and we work in the category of commutative $R$-algebras. For an abelian group $G$, we denote by $R G$ the group algebra of $G$ over $R$. Let $A$ be an $R$-algebra and consider the following two conditions.

(i) $A$ is generated by idempotents over $R$;

(ii) $A$ is a quotient ring of $R G$ for a torsion abelian group $G$ without an element whose order is a zero-divisor in $R$.

In [4], it has been shown that, if $R$ is an algebraically closed field, then the above two conditions are equivalent. However, as remarked in the paper, this is no longer valid if we drop the assumption that $R$ is an algebraically closed field; both implications (i) $\Rightarrow$ (ii) and (ii) $\Rightarrow$ (i) have counter-examples even if $R$ is a field. This paper was originally motivated by seeking conditions for $R$ to hold the equivalence of (i) and (ii) without any additional assumption on $A$.

This paper consists of four sections.

In Section 2, we collect some preliminary results that we need in later sections.

2010 AMS Mathematics subject classification. Primary 13A99, Secondary $16 \mathrm{~S} 34$.

Received by the editors on February 11, 2014, and in revised form on April 25, 2014. 
In Section 3, we are mainly interested in the case where the implication (ii) $\Rightarrow$ (i) always holds; namely, the case where every $R$-algebra $A$ satisfying (ii) also satisfies (i). Concerning this, we immediately know that (ii) $\Rightarrow$ (i) always holds if and only if $R G$ is generated by idempotents over $R$ for every torsion abelian group $G$ without an element whose order is a zero-divisor in $R$. So, we studied conditions for group algebras to be generated by idempotents in [6]. We apply the results obtained in [6] to prove that the following two conditions are equivalent (Proposition 3.2):

(1) The implication (ii) $\Rightarrow$ (i) always holds;

(2) $n$ is a unit of $R$ and the $n$th cyclotomic polynomial $\phi_{n}(X)$ has a root in $R$ for every positive integer $n$ that is not a zero-divisor in $R$.

We then turn to considering the reverse implication and, using similar arguments in $[4,5]$ we give a condition for $R$ to hold (i) $\Rightarrow$ (ii) without any assumption on $A$ (Proposition 3.4). Combining Proposition 3.2 with Proposition 3.4, we prove that, if there exists a prime number $p$ that is not a zero-divisor in $R$, then the above condition (2) is in fact necessary and sufficient for $R$ to hold the equivalence of (i) and (ii) (Theorem 3.6).

In studying algebras generated by idempotents, we frequently encounter $R$-algebras $A$ such that $A_{\text {red }}$ is generated by idempotents over $R$, but $A$ itself is not, where $A_{\text {red }}=A / \operatorname{nil}(A)$, the quotient ring of $A$ by its nilradical. In view of this, in Section 4 we take up the following conditions for $R$-algebras $A$.

(i') $A_{\text {red }}$ is generated by idempotents over $R$;

(ii') $A$ is a quotient ring of $R G$ for a torsion abelian group $G$.

We aim at considering a similar problem as in Section 3 and, after giving some results concerning the implications (i') $\Rightarrow$ (ii') and (ii') $\Rightarrow$ (i'), we prove that the equivalence of (i') and (ii') holds for $R$ if and only if the characteristic of $R$ is positive and $\phi_{n}(X)$ has a root in $R_{\text {red }}$ for every positive integer $n$ (Theorem 4.14).

We denote by $U(R)$ the group of units in $R$ and by $R[X]$ the polynomial ring in one indeterminate $X$ over $R$. The characteristic of $R$ is denoted by $\operatorname{ch}(R)$. We write $\mathbf{Z}, \mathbf{Q}, \mathbf{C}$ and $\mathbf{F}_{p}$ for the ring of integers, the field of rational numbers, the field of complex numbers 
and the prime field of characteristic $p>0$, respectively. If $R$-algebras $A$ and $B$ are isomorphic as $R$-algebras, we indicate this by $A \cong_{R} B$. When considering an integer $n$ as an element of $R$, we assume that $n$ stands for $n \cdot 1_{R}$, as usual. For basic results and undefined terminology, our general references are $[\mathbf{2}, \mathbf{3}, \mathbf{8}]$.

As noted above, we often use the following.

Lemma 1.1 ([6], Lemma 3.3). Let $n$ be a positive integer. Then the following conditions are equivalent:

(1) $R[X] /\left(X^{n}-1\right)$ is generated by idempotents over $R$.

(2) $n \in U(R)$ and $\phi_{n}(X)$ has a root $c$ in $R$.

Moreover, if $R$ is indecomposable, then the above conditions are also equivalent to the following:

(3) $n \in U(R)$ and $U(R)$ contains an element $c$ of order $n$.

Lemma 1.2 ([6], Corollary 3.5). Let $n$ be a positive integer, and write $n=p_{1}^{a_{1}} \cdots p_{m}^{a_{m}}$, where $p_{1}, \ldots, p_{m}$ are distinct prime numbers and $a_{1}, \ldots, a_{m}$ are positive integers. Let $q_{i}=p_{i}^{a_{i}}$ for each $i=1, \ldots, m$. Then the following conditions are equivalent:

(1) $\phi_{n}(X)$ has a root in $R$.

(2) $\phi_{q_{i}}(X)$ has a root in $R$ for each $i$.

Theorem 1.3 ([6], Theorem 4.2). Let $G$ be an abelian group. Then the following conditions are equivalent:

(1) $R G$ is generated by idempotents over $R$.

(2) $G$ is a torsion group with $\operatorname{supp} G \subseteq U(R)$, and $\phi_{p^{m}}(X)$ has a root in $R$ for every $p \in \operatorname{supp} G$ and positive integer $m$ with $p^{m} \leq e_{p}(G)$.

(3) $G$ is a torsion group with $\operatorname{supp} G \subseteq U(R)$, and $\phi_{n}(X)$ has a root in $R$ for every positive integer $n$ such that $n=$ ord $(g)$ for some $g \in G$.

Moreover, if $R$ is indecomposable, then the above conditions are also equivalent to the following:

(4) $G$ is a torsion group with $\operatorname{supp} G \subseteq U(R)$, and for every $g \in G$ there exists $c \in U(R)$ such that ord $(c)=$ ord $(g)$. 
In the rest of this paper, for an $R$-algebra $A$, we denote by $\Delta(A)$ the subalgebra of $A$ generated by the set of all idempotents over $R$.

2. Some preliminary results. In this section we collect some preliminary results that we need in later sections.

Lemma 2.1. Let $n$ be a positive integer, and write $n=p_{1}^{a_{1}} \cdots p_{m}^{a_{m}}$, where $p_{1}, \ldots, p_{m}$ are distinct prime numbers and $a_{1}, \ldots, a_{m}$ are positive integers. Let $\mathfrak{a}=0:_{R} n$ and $\mathfrak{a}_{i}=0:_{R} p_{i}^{a_{i}}$ for each $i$. Then the following conditions are equivalent:

(1) $n \in U(R / \mathfrak{a})$.

(2) $R \cong_{R} R / \mathfrak{a} \times R / n R$.

(3) $p_{i} \in U\left(R / \mathfrak{a}_{i}\right)$ for each $i$.

(4) $R \cong_{R} R / \mathfrak{a}_{i} \times R / p_{i}^{a_{i}} R$ for each $i$.

(5) $n \in n^{2} R$.

Moreover, if $\operatorname{ch}(R)=0$, then the above conditions are also equivalent to the following condition:

(6) There is a subring of $R$ isomorphic to $\mathbf{Z}\left[n^{-1}\right] \times \mathbf{Z} / d \mathbf{Z}$ for some divisor $d$ of $n$.

Proof. We set $q_{i}=p_{i}^{a_{i}}$ and $n_{i}=n / q_{i}$ for each $i$. First of all, we note that

$$
\mathfrak{a}=\mathfrak{a}_{i}+\left(0:_{R} n_{i}\right) \subseteq \mathfrak{a}_{i}+q_{i} R
$$

for each $i$. In fact, let $s_{i}$ and $t_{i}$ be integers such that $n_{i} s_{i}+q_{i} t_{i}=1$. Then $\mathfrak{a}=n_{i} s_{i} \mathfrak{a}+q_{i} t_{i} \mathfrak{a}$, where $n_{i} s_{i} \mathfrak{a} \subseteq \mathfrak{a}_{i}$ and $q_{i} t_{i} \mathfrak{a} \subseteq\left(\begin{array}{lll}0: R & n_{i}\end{array}\right)$. Thus, $\mathfrak{a} \subseteq \mathfrak{a}_{i}+\left(0:_{R} n_{i}\right)$. Since the reverse containment is obvious, we know that the equality in $(2.1)$ holds. Moreover $q_{i} t_{i} \mathfrak{a} \subseteq q_{i} R$, so that $\mathfrak{a} \subseteq \mathfrak{a}_{i}+q_{i} R$ also holds.

$(1) \Leftrightarrow(2)$. We have $n \in U(R / \mathfrak{a})$ if and only if $\mathfrak{a}+n R=R$, so that (1) is equivalent to (2), because $n \mathfrak{a}=0$.

$(3) \Leftrightarrow(4)$. This is an immediate consequence of the equivalence (1) $\Leftrightarrow(2)$.

$(2) \Rightarrow(4)$. Since $R \cong_{R} R / \mathfrak{a} \times R / n R$, it follows that $\mathfrak{a}+n R=R$, and hence $\mathfrak{a}_{i}+q_{i} R=R$ by (2.1). Thus, $R \cong_{R} R / \mathfrak{a}_{i} \times R / q_{i} R$, because $q_{i} \mathfrak{a}_{i}=0$. 
$(4) \Rightarrow(2)$. From (2.1), we have

$$
\mathfrak{a}=\mathfrak{a}_{1}+\cdots+\mathfrak{a}_{m}
$$

by induction on $m$. Since $\mathfrak{a}_{i}+q_{i} R=R$ for each $i$, it thus follows that

$$
\mathfrak{a}+n R \supseteq\left(\mathfrak{a}_{1}+q_{1} R\right) \cdots\left(\mathfrak{a}_{m}+q_{m} R\right)=R,
$$

and therefore $\mathfrak{a}+n R=R$. Thus, $R \cong_{R} R / \mathfrak{a} \times R / n R$.

$(1) \Leftrightarrow(5)$. This is easily verified.

$(2) \Rightarrow(6)$. Note that $\mathfrak{a} \cap \mathbf{Z}=0$, because $\operatorname{ch}(R)=0$. Since $n \in U(R / \mathfrak{a})$, it follows that $\mathbf{Z}\left[n^{-1}\right] \subseteq R / \mathfrak{a}$. Moreover we have $n R \cap \mathbf{Z}=d \mathbf{Z}$ for some divisor $d$ of $n$, so that $\mathbf{Z} / d \mathbf{Z} \subseteq R / n R$. Thus, (6) holds.

$(6) \Rightarrow(2)$. Let $e_{1}=\left(n^{-1}, 0\right)$ and $e_{2}=(0,1)$, which are elements in $\mathbf{Z}\left[n^{-1}\right] \times \mathbf{Z} / d \mathbf{Z}$. Then $n e_{2}=0$, and hence $e_{2} \in \mathfrak{a}$. Since $n e_{1}+e_{2}=1$, it thus follows that $n R+\mathfrak{a}=R$, which implies (2).

Lemma 2.2. Let $A=R G$, where $G$ is a cyclic group of order $n$. Write $n=p_{1}^{a_{1}} \cdots p_{m}^{a_{m}}$ with distinct prime numbers $p_{1}, \ldots, p_{m}$ and positive integers $a_{1}, \ldots, a_{m}$. Then the following conditions are equivalent:

(1) $A_{\text {red }}=\Delta\left(A_{\text {red }}\right)$.

(2) $p_{i} \in p_{i}^{2} R_{\text {red }}$ and $\phi_{q_{i}}(X)$ has a root in $R_{\text {red }}$ for each $i$, where $q_{i}=p_{i}^{a_{i}}$.

(3) $n \in n^{2} R_{\text {red }}$ and $\phi_{n}(X)$ has a root in $R_{\text {red }}$.

Proof. Set $\mathfrak{a}_{i}=0: R_{\text {red }} p_{i}^{a_{i}}$ for each $i$. Noting that $\mathfrak{a}_{i}=0: R_{\text {red }} p_{i}$, we can say that $p_{i} \in U\left(R_{\text {red }} / \mathfrak{a}_{i}\right)$ if and only if $p_{i} \in p_{i}^{2} R_{\text {red }}$ for every $p_{i}$ by Lemma 2.1. Hence, the equivalence $(2) \Leftrightarrow(3)$ follows from Lemma 2.1 and Lemma 1.2. For $(1) \Leftrightarrow(2)$, we note the following identity: $\phi_{p^{e}}(X)=\phi_{p^{e-1}}\left(X^{p}\right)$ with a prime number $p$ and a positive integer $e$. From this, if $\phi_{p^{e}}(X)$ has a root in $R_{\text {red }}$, then $\phi_{p^{t}}(X)$ has a root in $R_{\text {red }}$ for every positive integer $t \leq e$. Hence, the equivalence (1) $\Leftrightarrow(2)$ follows from [7, Theorem 2.12].

As shown in [7, Remark 2.14], if $\operatorname{ch}(R)>0$, then $n \in n^{2} R_{\text {red }}$ for every integer $n$. Thus, we have 
Corollary 2.3. Let $A=R G$, where $G$ is a cyclic group of order $n$, and suppose that $\operatorname{ch}(R)>0$. Then the following conditions are equivalent:

(1) $A_{\text {red }}=\Delta\left(A_{\text {red }}\right)$.

(2) $\phi_{n}(X)$ has a root in $R_{\text {red }}$.

Lemma 2.4. For a positive integer n, we set

$$
\theta_{n}(X)=X^{n-1}+X^{n-2}+\cdots+X+1 .
$$

Suppose that there exist integers $n_{1}, \ldots, n_{m}$ and elements $c_{1}, \ldots, c_{m} \in$ $R$ such that $\theta_{n_{i}}\left(c_{i}\right)=0$ for each $i$. Then $c_{i}^{n_{i}}=1$ for each $i$. Moreover, if the greatest common divisor (g.c.d.) of $n_{1}, \ldots, n_{m}$ belongs to $U(R)$, then $R\left(1-c_{1}\right)+\cdots+R\left(1-c_{m}\right)=R$.

Proof. The assertion that $c_{i}^{n_{i}}=1$ is obvious, because

$$
1-c_{i}^{n_{i}}=\left(1-c_{i}\right) \theta_{n_{i}}\left(c_{i}\right)=0 .
$$

Let $d$ be the g.c.d. of $n_{1}, \ldots, n_{m}$, and suppose that $d \in U(R)$. Since $d \in \mathbf{Z} n_{1}+\cdots+\mathbf{Z} n_{m}$ and

$$
n_{i}=n_{i}-\theta_{n_{i}}\left(c_{i}\right)=\left(1-c_{i}\right)+\cdots+\left(1-c_{i}^{n_{i}-1}\right) \in R\left(1-c_{i}\right)
$$

for each $i$, it then follows that

$$
d \in R n_{1}+\cdots+R n_{m} \subseteq R\left(1-c_{1}\right)+\cdots+R\left(1-c_{m}\right),
$$

and therefore $R\left(1-c_{1}\right)+\cdots+R\left(1-c_{m}\right)=R$, because $d \in U(R)$.

3. Algebras generated by idempotents. In what follows, groups are assumed to be multiplicative, namely, the operation of a group is written multiplicatively. The order of an element $g$ of a group is denoted by ord $(g)$.

Before proceeding further, we recall some notion concerning abelian groups. Let $G$ be an abelian group. Then, we set

$$
G_{t}=\{g \in G \mid \operatorname{ord}(g)<\infty\},
$$

which is a subgroup called the torsion part of $G$. For a prime number $p$, we set

$$
e_{p}(G)=\sup \left\{\operatorname{ord}(g) \mid g \in G \text { and } \quad \text { ord }(g)=p^{n} \text { for some } n \geq 0\right\},
$$


so that $1 \leq e_{p}(G) \leq \infty$. If $G$ is a finite group, then we set

$$
e(G)=\max \{\operatorname{ord}(g) \mid g \in G\},
$$

and call $e(G)$ the exponent of $G$. Finally we set

$$
\operatorname{supp} G=\left\{p \mid e_{p}(G) \neq 1\right\},
$$

which is called the support of $G$. Note that $\operatorname{supp} G=\emptyset$, the empty set, if and only if $G_{t}$ is the trivial group.

We say that an integer $n$ is $R$-regular if $n$ is not a zero-divisor in $R$. Let $G$ be a non-trivial torsion abelian group such that $R G=$ $\Delta(R G)$. Then it follows from Theorem 1.3 that supp $G \subseteq U(R)$, and in particular, every $p \in \operatorname{supp} G$ is $R$-regular. With this in mind, letting

$$
\Gamma(R)=\{n \mid n \text { is an } R \text {-regular positive integer }\}
$$

and

$$
\Gamma_{0}(R)=\{p \mid p \text { is a prime number contained in } \Gamma(R)\},
$$

we consider the following two conditions for $R$-algebras $A$ :

(i) $A$ is generated by idempotents over $R$;

(ii) $A$ is a quotient ring of $R G$ for a torsion abelian group $G$ satisfying $\operatorname{supp} G \subseteq \Gamma_{0}(R)$.

As mentioned in the introduction, the original motivation of this paper is seeking conditions for $R$ to hold the equivalence of (i) and (ii). As an application of Theorem 1.3, we give a complete answer to this problem. For a precise description of our results, let $\mathcal{C}_{\text {id }}(R)$ be the class of $R$-algebras $A$ satisfying condition (i) and $\mathcal{C}_{g r}(R)$ the class of $R$-algebras $A$ satisfying condition (ii). We use set-theoretical notation for these classes; for example, we write $A \in \mathcal{C}_{\text {id }}(R)$ to mean that $A$ is an $R$-algebra generated by idempotents over $R$. Our purpose is thus to give a condition for $R$ to hold the equality $\mathcal{C}_{\text {id }}(R)=\mathcal{C}_{g r}(R)$.

\section{Remark 3.1.}

(1) For an integer $n>1$, we have $n \in \Gamma(R)$ if and only if $p \in \Gamma_{0}(R)$ for every prime divisor $p$ of $n$. In particular, we have $\Gamma(R)=\{1\}$ if and only if $\Gamma_{0}(R)=\emptyset$, and $\Gamma(R) \subseteq U(R)$ if and only if $\Gamma_{0}(R) \subseteq U(R)$. 
(2) $\Gamma_{0}(R)$ can be the empty set. For example, let $p_{1}=2, p_{2}=3$, $p_{3}=5, \ldots$ be the sequence of all the prime numbers, and set

$$
R=\mathbf{Z}\left[X_{1}, X_{2}, X_{3}, \ldots\right] /\left(p_{1} X_{1}, p_{2} X_{2}, p_{3} X_{3}, \ldots\right),
$$

where $X_{1}, X_{2}, X_{3}, \ldots$ are indeterminates. Then, clearly, we have $\Gamma_{0}(R)=\emptyset$. Note that $\Gamma_{0}(R)=\emptyset$ implies $\operatorname{ch}(R)=0$ because, if $\operatorname{ch}(R)=n>0$, then

$$
\Gamma_{0}(R)=\{p \mid p \text { is a prime number not dividing } n\},
$$

which is a non-empty subset of $U(R)$.

(3) A torsion abelian group $G$ satisfies $\operatorname{supp} G \subseteq \Gamma_{0}(R)$ if and only if ord $(g)$ is $R$-regular for every $g \in G$. In particular, if $G$ is generated by $\left\{g_{\lambda} \mid \lambda \in \Lambda\right\}$ and, if $\operatorname{ord}\left(g_{\lambda}\right)$ is $R$-regular for every $\lambda \in \Lambda$, then $\operatorname{supp} G \subseteq \Gamma_{0}(R)$.

(4) For an $R$-algebra $A$, we have $A \in \mathcal{C}_{g r}(R)$ if and only if there exists a torsion subgroup $G$ of $U(A)$ such that $\operatorname{supp} G \subseteq \Gamma_{0}(R)$ and $A=R[G]$. Indeed, suppose that there exists such a subgroup $G$ of $U(A)$. Then we can define a surjective $R$-algebra homomorphism $\sigma: R G \rightarrow A$ by $\sigma(g)=g$ for $g \in G$, and hence $A \in \mathcal{C}_{g r}(R)$. Conversely, suppose that $A \in \mathcal{C}_{g r}(R)$, and let $\sigma: R G \rightarrow A$ be a surjective $R$-algebra homomorphism, where $G$ is a torsion abelian group with supp $G \subseteq \Gamma_{0}(R)$. Since $G \subseteq U(R G)$, it then follows that $\sigma(G)$ is a torsion subgroup of $U(A)$ such that $\operatorname{supp} \sigma(G) \subseteq \Gamma_{0}(R)$ and $A=R[\sigma(G)]$.

Using Theorem 1.3, we first give a condition for $R$ to hold $\mathcal{C}_{\text {id }}(R) \supseteq$ $\mathcal{C}_{g r}(R)$. Note that $R G \in \mathcal{C}_{g r}(R)$ for every torsion abelian group $G$ satisfying that $\operatorname{supp} G \subseteq \Gamma_{0}(R)$.

Proposition 3.2. The following conditions are equivalent:

(1) $\mathcal{C}_{\text {id }}(R) \supseteq \mathcal{C}_{g r}(R)$.

(2) $\Gamma(R) \subseteq U(R)$ and $\phi_{n}(X)$ has a root in $R$ for every $n \in \Gamma(R)$.

(3) $\Gamma_{0}(R) \subseteq U(R)$ and $\phi_{p^{m}}(X)$ has a root in $R$ for every $p \in \Gamma_{0}(R)$ and positive integer $m$.

Proof. The equivalence of (2) and (3) follows from Lemma 1.2 and Remark 3.1 (1). Suppose that $\Gamma_{0}(R)=\emptyset$. Then (3) holds, because there exist no elements in $\Gamma_{0}(R)$. On the other hand, (1) also holds. 
Indeed, the trivial group is the only torsion abelian group with support contained in $\Gamma_{0}(R)$, and therefore it follows that

$$
\mathcal{C}_{g r}(R)=\{R / I \mid I \text { is an ideal of } R\} .
$$

Since $R / I \in \mathcal{C}_{\text {id }}(R)$ for every ideal $I$ of $R$, we surely have $\mathcal{C}_{\text {id }}(R) \supseteq$ $\mathcal{C}_{g r}(R)$. Hence, the equivalence of (1) and (3) trivially holds in this case. Thus, we may assume that $\Gamma_{0}(R) \neq \emptyset$.

$(1) \Rightarrow(3)$. Let $p$ be an element of $\Gamma_{0}(R)$, and let $m$ be a positive integer. Then, letting $G$ be a cyclic group of order $p^{m}$, we have $\operatorname{supp} G=\{p\} \subseteq \Gamma_{0}(R)$, and hence $R G \in \mathcal{C}_{g r}(R)$. Since $\mathcal{C}_{\text {id }}(R) \supseteq$ $\mathcal{C}_{g r}(R)$, it follows that $R G \in \mathcal{C}_{\text {id }}(R)$, i.e., $R G=\Delta(R G)$. Thus, $p \in U(R)$ and $\phi_{p^{m}}(X)$ has a root in $R$ by virtue of Theorem 1.3.

$(3) \Rightarrow(1)$. Let $A$ be an $R$-algebra such that $A \in \mathcal{C}_{g r}(R)$, so that there exists a surjective $R$-algebra homomorphism $R G \rightarrow A$ for a torsion abelian group $G$ satisfying that supp $G \subseteq \Gamma_{0}(R)$. Since (3) holds, it then follows from Theorem 1.3 that $R G=\Delta(R G)$, which implies $A=\Delta(A)$, i.e., $A \in \mathcal{C}_{\text {id }}(R)$. Therefore, $\mathcal{C}_{\text {id }}(R) \supseteq \mathcal{C}_{g r}(R)$, as desired.

Remark 3.3. The proof of the above proposition shows that if $\Gamma_{0}(R)=$ $\emptyset$, then $\mathcal{C}_{\text {id }}(R) \neq \mathcal{C}_{g r}(R)$; indeed, we have $R^{2} \in \mathcal{C}_{\text {id }}(R)$, while $R^{2} \notin$ $\mathcal{C}_{g r}(R)$ by virtue of $(3.1)$.

We now turn to consideration of the reverse inclusion $\mathcal{C}_{\text {id }}(R) \subseteq$ $\mathcal{C}_{g r}(R)$.

Proposition 3.4. The following conditions are equivalent:

(1) $\mathcal{C}_{\text {id }}(R) \subseteq \mathcal{C}_{g r}(R)$

(2) $R^{2} \in \mathcal{C}_{g r}(R)$.

(3) There exist torsion elements $c_{1}, \ldots, c_{m}$ in $U(R)$ such that ord $\left(c_{i}\right)$ is an $R$-regular integer for each $i$ and $R\left(1-c_{1}\right)+\cdots+R\left(1-c_{m}\right)=R$.

Proof. Since the implication $(1) \Rightarrow(2)$ is obvious, we prove $(2) \Rightarrow$ (3) and $(3) \Rightarrow(1)$.

$(2) \Rightarrow(3)$. Since $R^{2} \in \mathcal{C}_{g r}(R)$, there exists a torsion subgroup $G$ of $U\left(R^{2}\right)=U(R) \times U(R)$ such that $\operatorname{supp} G \subseteq \Gamma_{0}(R)$ and $R^{2}=R[G]$. 
Note that, for $f \in R[G]$, we can write $f=a_{1} g_{1}+\cdots+a_{m} g_{m}$, where $a_{1}, \ldots, a_{m} \in R$ and $g_{1}, \ldots, g_{m} \in G$. Hence, we have

$$
R^{2} \ni(1,0)=a_{1}\left(u_{1}, v_{1}\right)+\cdots+a_{m}\left(u_{m}, v_{m}\right)
$$

for some $a_{1}, \ldots, a_{m} \in R$ and $\left(u_{1}, v_{1}\right), \ldots,\left(u_{m}, v_{m}\right) \in G$. Let $b_{i}=a_{i} u_{i}$ and $c_{i}=u_{i}^{-1} v_{i}$ for every $i$. Then each ord $\left(c_{i}\right)$ is an $R$-regular integer, because $\operatorname{supp} G \subseteq \Gamma_{0}(R)$. Moreover, it follows from (3.2) that $b_{1}+\cdots+b_{m}=1$ and $b_{1} c_{1}+\cdots+b_{m} c_{m}=0$, and hence $b_{1}\left(1-c_{1}\right)+\cdots+b_{m}\left(1-c_{m}\right)=1$. Therefore, $R\left(1-c_{1}\right)+\cdots+R\left(1-c_{m}\right)=$ $R$.

$(3) \Rightarrow(1)$. Let $n_{i}=\operatorname{ord}\left(c_{i}\right)$ for each $i$, and let $a_{1}, \ldots, a_{m}$ be elements in $R$ such that

$$
a_{1}\left(1-c_{1}\right)+\cdots+a_{m}\left(1-c_{m}\right)=1 .
$$

We set $a=a_{1}+\cdots+a_{m}$ and $b=a_{1} c_{1}+\cdots+a_{m} c_{m}$, so that $a-b=1$. Now let $A$ be an $R$-algebra generated by idempotents over $R$. Then there exists a set $E=\left\{e_{\lambda} \mid \lambda \in \Lambda\right\}$ of idempotents in $A$ such that $A=R[E]$. Let

$$
g_{\lambda, i}=e_{\lambda}+c_{i}\left(1-e_{\lambda}\right)
$$

for $\lambda \in \Lambda$ and $i=1, \ldots, m$. Then

$$
g_{\lambda, i}{ }^{n_{i}}=e_{\lambda}+c_{i}^{n_{i}}\left(1-e_{\lambda}\right)=1,
$$

and hence each $g_{\lambda, i}$ is an element of $U(A)$ such that ord $\left(g_{\lambda, i}\right)$ is an $R$-regular integer, because ord $\left(g_{\lambda, i}\right)$ is a divisor of $n_{i}$ and $n_{i}$ is $R$-regular. Thus, letting $G$ be the subgroup of $U(A)$ generated by $\left\{g_{\lambda, i} \mid \lambda \in \Lambda, 1 \leq i \leq m\right\}$, we know that $G$ is a torsion group satisfying $\operatorname{supp} G \subseteq \Gamma_{0}(R)$. Moreover, for each $\lambda \in \Lambda$, we have

$$
a_{1} g_{\lambda, 1}+\cdots+a_{m} g_{\lambda, m}-b=a e_{\lambda}+b\left(1-e_{\lambda}\right)-b=e_{\lambda},
$$

which implies $A=R[G]$. Therefore, $A \in \mathcal{C}_{g r}(R)$, i.e., $\mathcal{C}_{\text {id }}(R) \subseteq$ $\mathcal{C}_{g r}(R)$.

Corollary 3.5. Let $\theta_{n}(X)$ be the polynomial defined in Lemma 2.4. If there exist $R$-regular integers $n_{1}, \ldots, n_{m}$ such that their g.c.d. belongs to $U(R)$ and $\theta_{n_{i}}(X)$ has a root in $R$ for each $i$, then $\mathcal{C}_{\mathrm{id}}(R) \subseteq \mathcal{C}_{g r}(R)$.

In particular, if there exists a prime number $p$ such that $p \in U(R)$ and $\phi_{p}(X)$ has a root in $R$, then $\mathcal{C}_{\mathrm{id}}(R) \subseteq \mathcal{C}_{g r}(R)$. 
Proof. For every $i$, let $c_{i} \in R$ be an element such that $\theta_{n_{i}}\left(c_{i}\right)=0$. Then $c_{i}^{n_{i}}=1$, and hence each $c_{i}$ is a torsion element of $U(R)$ such that ord $\left(c_{i}\right)$ is an $R$-regular integer. Moreover we have $R\left(1-c_{1}\right)+$ $\cdots+R\left(1-c_{m}\right)=R$ by Lemma 2.4 , and therefore $\mathcal{C}_{\text {id }}(R) \subseteq \mathcal{C}_{g r}(R)$ by Proposition 3.4.

We are now ready to state and prove the following:

Theorem 3.6. The following conditions are equivalent:

(1) $\mathcal{C}_{\text {id }}(R)=\mathcal{C}_{g r}(R)$

(2) $\Gamma(R) \neq \emptyset$ and $\mathcal{C}_{\text {id }}(R) \supseteq \mathcal{C}_{g r}(R)$.

(3) $\emptyset \neq \Gamma(R) \subseteq U(R)$ and $\phi_{n}(X)$ has a root in $R$ for every $n \in \Gamma(R)$.

(4) $\emptyset \neq \Gamma_{0}(R) \subseteq U(R)$ and $\phi_{p^{m}}(X)$ has a root in $R$ for every $p \in \Gamma_{0}(R)$ and positive integer $m$.

Moreover, if $R$ is indecomposable, then the above conditions are also equivalent to the following:

(5) $\emptyset \neq \Gamma_{0}(R) \subseteq U(R)$ and $U(R)$ contains an element of order $p^{m}$ for every $p \in \Gamma_{0}(R)$ and positive integer $m$.

Proof. The implication (1) $\Rightarrow(2)$ is obvious (cf., Remark 3.3), and the equivalence of (2), (3) and (4) is an immediate consequence of Proposition 3.2. Suppose that (4) holds. Then $\mathcal{C}_{\text {id }}(R) \subseteq \mathcal{C}_{g r}(R)$ by Corollary 3.5, while $\mathcal{C}_{\text {id }} \supseteq \mathcal{C}_{g r}(R)$ because of the implication $(4) \Rightarrow(2)$. Thus, $\mathcal{C}_{\text {id }}(R)=\mathcal{C}_{g r}(R)$, namely, (1) holds. Finally, the equivalence of (4) and (5) follows from Lemma 1.1.

Remark 3.7. Concerning the above theorem, the condition $\mathcal{C}_{\text {id }}(R) \subseteq$ $\mathcal{C}_{g r}(R)$ is not equivalent to the condition $\mathcal{C}_{\text {id }}(R)=\mathcal{C}_{g r}(R)$ in general. Indeed, let $R=\mathbf{F}_{2}[X] /\left(X^{2}+X+1\right)$. Then $\phi_{3}(X)$ has a root in $R$, so that $\mathcal{C}_{\text {id }}(R) \subseteq \mathcal{C}_{g r}(R)$ by Corollary 3.5. However, let $G$ be a cyclic group of order 5 . Then $R G \in \mathcal{C}_{g r}(R)$, while $R G \notin \mathcal{C}_{\text {id }}(R)$ by Theorem 1.3, because $\phi_{5}(X)$ does not have a root in $R$. Thus, $\mathcal{C}_{\text {id }}(R) \neq \mathcal{C}_{g r}(R)$.

4. Algebras whose quotient rings by nilradicals are generated by idempotents. We continue the consideration of the relationship between algebras generated by idempotents and algebras 
that are quotient rings of group algebras. In studying algebras generated by idempotents, we frequently encounter $R$-algebras $A$ such that $A_{\text {red }}=\Delta\left(A_{\text {red }}\right)$, but $A \neq \Delta(A)$. It is thus natural to consider the class of $R$-algebras $A$ satisfying the following condition (i'), instead of (i):

(i') $A_{\text {red }}$ is generated by idempotents over $R$.

Let $\mathcal{C}_{\text {id }}^{\prime}(R)$ be the class of $R$-algebras $A$ satisfying the condition (i'), so that $\mathcal{C}_{\text {id }}(R) \subseteq \mathcal{C}_{\text {id }}^{\prime}(R)$, and $A \in \mathcal{C}_{\text {id }}^{\prime}(R)$ means $A_{\text {red }} \in \mathcal{C}_{i d}(R)$. Hence, by the results obtained in the preceding section, we know conditions for $A \in \mathcal{C}_{\text {id }}^{\prime}(R)$ to hold $A_{\text {red }} \in \mathcal{C}_{g r}(R)$. However, $A_{\text {red }} \in \mathcal{C}_{g r}(R)$ does not imply $A \in \mathcal{C}_{g r}(R)$, in general, as the following example shows.

Example 4.1. Let $R=\mathbf{F}_{p}$, and let $A=R[X] /\left(X^{p}-1\right)$. Then $A_{\text {red }}=R$, which obviously belongs to $\mathcal{C}_{g r}(R)$. However $A \notin \mathcal{C}_{g r}(R)$. Indeed, note that $f^{p} \in \mathbf{F}_{p}$ for any $f \in A$, so that $f^{p(p-1)}$ is either 1 or 0 . It thus follows that $U(A)^{p(p-1)}=1$. Moreover, letting $f \in U(A)$ be an element such that $f^{p-1}=1$, we have $f=f^{p} \in \mathbf{F}_{p}$. Hence, if $G$ is a torsion abelian group for which there exists a surjection $\sigma: R G \rightarrow A$, then $G$ must contain an element $g$ such that $p=$ ord $(\sigma(g))$. For such $g$ we have $p \mid \operatorname{ord}(g)$, so that $p \in \operatorname{supp} G$. Thus, $G$ does not satisfy that $\operatorname{supp} G \subseteq \Gamma_{0}(R)$. Note that $A \cong_{R} R C_{p}$, where $C_{p}$ denotes the cyclic group of order $p$.

In view of this, corresponding to (i') we consider the following condition, which is a generalization of (ii).

(ii') $A$ is a quotient ring of $R G$ for a torsion abelian group $G$.

Let $\mathcal{C}_{g r}^{\prime}(R)$ be the class of $R$-algebras $A$ satisfying the condition (ii'). The purpose of this section is to give a condition for $R$ to hold the equality $\mathcal{C}_{\mathrm{id}}^{\prime}(R)=\mathcal{C}_{g r}^{\prime}(R)$.

\section{Remark 4.2.}

(1) For an $R$-algebra $A$, we have $A \in \mathcal{C}_{g r}^{\prime}(R)$ if and only if there exists a torsion subgroup $G$ of $U(A)$ such that $A=R[G]$ (cf., Remark $3.1(4))$.

(2) Suppose that $A \in \mathcal{C}_{g r}^{\prime}(R)$ and that $A$ is finitely generated over $R$. Then $A$ is a quotient ring of $R G$ for some finite abelian group $G$. Indeed, since $A \in \mathcal{C}_{g r}^{\prime}(R)$, we have $A=R\left[G^{\prime}\right]$ for a torsion 
subgroup $G^{\prime}$ of $U(A)$. Then there exists a finite subset $H$ of $G^{\prime}$ such that $A=R[H]$, because $A$ is finitely generated over $R$. Let $G=\langle H\rangle$, where $\langle H\rangle$ denotes the subgroup of $G^{\prime}$ generated by the elements in $H$. Note that $G$ is a finite group, because $G$ is a finitely generated subgroup of the torsion abelian group $G^{\prime}$. Since $A=R[G]$, it follows that $A$ is a quotient ring of $R G$, which proves the assertion.

First we consider the case where the implication $\mathcal{C}_{g r}^{\prime}(R) \subseteq \mathcal{C}_{\text {id }}^{\prime}(R)$ holds.

Lemma 4.3. The following conditions are equivalent:

(1) $\mathcal{C}_{g r}^{\prime}(R) \subseteq \mathcal{C}_{\mathrm{id}}^{\prime}(R)$

(2) $(R G)_{\text {red }}=\Delta\left((R G)_{\text {red }}\right)$ for every finite cyclic group $G$.

Proof. Since $R G \in \mathcal{C}_{g r}^{\prime}(R)$ for every torsion abelian group $G$, the implication $(1) \Rightarrow(2)$ is obvious. For the reverse implication, it suffices to show that $(R G)_{\text {red }}=\Delta\left((R G)_{\text {red }}\right)$ for every torsion abelian group $G$. Indeed, if $A \in \mathcal{C}_{g r}^{\prime}(R)$, then $A$ is a surjective image of $R G$ for a torsion abelian group $G$, which implies $A_{\text {red }}$ is a surjective image of $(R G)_{\text {red }}$; thus, $A_{\text {red }}=\Delta\left(A_{\text {red }}\right)$ if $(R G)_{\text {red }}=\Delta\left((R G)_{\text {red }}\right)$. Let $\tau: R G \rightarrow(R G)_{\text {red }}$ be the natural $R$-algebra homomorphism, and let $\bar{G}=\tau(G)$. Then $(R G)_{\mathrm{red}}=R[\bar{G}]$. For $g \in G$, we set $\bar{g}=\tau(g)$. Since $R\langle g\rangle_{\mathrm{red}}=\Delta\left(R\langle g\rangle_{\mathrm{red}}\right)$, there exists a set $E_{g}$ of idempotents in $R\langle g\rangle_{\mathrm{red}}$ such that $R\langle g\rangle_{\text {red }}=R\left[E_{g}\right]$. Note that $R\langle g\rangle_{\text {red }} \subseteq(R G)_{\text {red }}$, so that $E_{g}$ is a subset of $(R G)_{\text {red }}$. We set $E=\cup_{g \in G} E_{g}$. Since $\bar{g} \in R\langle g\rangle_{\text {red }}$, it then follows that

$$
\bar{g} \in R\left[E_{g}\right] \subseteq R[E],
$$

which implies $\bar{G} \subseteq R[E]$. Thus, $R[\bar{G}] \subseteq R[E]$, and hence $(R G)_{\text {red }}=$ $R[E]$. Therefore, $(R G)_{\text {red }}=\Delta\left((R G)_{\text {red }}\right)$, which completes the proof.

Proposition 4.4. Suppose that $\operatorname{ch}(R)>0$. Then the following conditions are equivalent:

(1) $\mathcal{C}_{g r}^{\prime}(R) \subseteq \mathcal{C}_{\mathrm{id}}^{\prime}(R)$

(2) $\phi_{n}(X)$ has a root in $R_{\text {red }}$ for every positive integer $n$. 
(3) $\phi_{p^{m}}(X)$ has a root in $R_{\text {red }}$ for every prime number $p$ and positive integer $m$.

Proof. The equivalence of (1) and (2) is an immediate consequence of Lemma 4.3 and Corollary 2.3, while the equivalence of (2) and (3) follows from Lemma 1.2.

Proposition 4.5. Suppose that $\operatorname{ch}(R)=0$. Then the following conditions are equivalent:

(1) $\mathcal{C}_{g r}^{\prime}(R) \subseteq \mathcal{C}_{\text {id }}^{\prime}(R)$.

(2) $n \in n^{2} R_{\text {red }}$ and $\phi_{n}(X)$ has a root in $R_{\text {red }}$ for every positive integer $n$.

(3) $\phi_{p^{m}}(X)$ has a root in $R_{\text {red }}$ for every prime number $p$ and positive integer $m$, and for every prime number $p$, either (a) or (b) below holds:

(a) $\mathbf{Z}\left[p^{-1}\right] \subseteq R_{\text {red }}$, i.e., $p \in U\left(R_{\text {red }}\right)$;

(b) $\mathbf{Z}\left[p^{-1}\right] \times \mathbf{F}_{p} \subseteq R_{\text {red }}$.

Proof. The equivalence of (1) and (2) follows from Lemmas 4.3 and 2.2 , while the equivalence of (2) and (3) follows from Lemmas 2.1 and 1.2 .

Next, we consider the case where $\mathcal{C}_{\text {id }}^{\prime}(R) \subseteq \mathcal{C}_{g r}^{\prime}(R)$ holds. Let $A$ be an $R$-algebra, and let $\pi: A \rightarrow A_{\text {red }}$ be the natural $R$-algebra map. Recall that $\pi$ induces a surjective group homomorphism $\pi: U(A) \rightarrow U\left(A_{\text {red }}\right)$, namely, $U\left(A_{\text {red }}\right) \cong U(A) /(1+\operatorname{nil}(A))$. For the torsion parts of $U(A)$ and $U\left(A_{\text {red }}\right)$, we have the following:

Lemma 4.6. If $\operatorname{ch}(A)>0$, then $1+\operatorname{nil}(A)$ is a torsion subgroup of $U(A)$. In particular, $U\left(A_{\text {red }}\right)_{t} \cong U(A)_{t} /(1+\operatorname{nil}(A))$.

Proof. Let $n=\operatorname{ch}(A)$ and write $n=p_{1}^{a_{1}} \cdots p_{m}^{a_{m}}$, where $p_{1}, \ldots, p_{m}$ are distinct prime numbers and $a_{1}, \ldots, a_{m}$ are positive integers. Then

$$
\mathbf{Z} / n \mathbf{Z} \cong \mathbf{Z} / p_{1}^{a_{1}} \mathbf{Z} \times \cdots \times \mathbf{Z} / p_{m}^{a_{m}} \mathbf{Z}
$$

which implies

$$
A \cong A \otimes \mathbf{Z}(\mathbf{Z} / n \mathbf{Z}) \cong A_{1} \times \cdots \times A_{m},
$$


where $A_{i}=A / p_{i}^{a_{i}} A$ for each $i$. It thus suffices to show that $1+\operatorname{nil}\left(A_{i}\right)$ is a torsion subgroup of $U\left(A_{i}\right)$ for each $i$. Hence, we may assume that $n=p^{a}$, a power of a prime number $p$. Now let $u$ be an element of $\operatorname{nil}(A)$, and take $r$ to satisfy $u^{p^{r}}=0$. Note that

$$
\left(\begin{array}{c}
p^{a+r-1} \\
i
\end{array}\right) \equiv 0 \quad\left(\bmod p^{a}\right)
$$

for every $i<p^{r}$. Thus, letting $q=p^{a+r-1}$, we have $(1+u)^{q}=1$, and hence $1+u$ is a torsion element of $U(A)$.

Lemma 4.7. Let $A$ be an $R$-algebra such that $A_{\mathrm{red}}=R[\bar{G}]$ for $a$ subgroup $\bar{G}$ of $U\left(A_{\text {red }}\right)$. Then there exists a subgroup $G$ of $U(A)$ such that $A=R[G]$. Suppose, in addition, that $\bar{G}$ is a torsion group and $\operatorname{ch}(R)>0$. Then we can take the group $G$ to be a torsion group.

Proof. Let $\pi: A \rightarrow A_{\text {red }}$ be the natural $R$-algebra homomorphism. Since $U\left(A_{\text {red }}\right) \cong U(A) /(1+\operatorname{nil}(A))$, we have $\bar{G} \cong G /(1+\operatorname{nil}(A))$ for some subgroup $G$ of $U(A)$. Then nil $(A) \subseteq R[G]$, because $1+\operatorname{nil}(A) \subseteq$ $G$. Since $\pi(R[G])=R[\bar{G}]=A_{\text {red }}=\pi(A)$, it thus follows that $A=R[G]$. If $\operatorname{ch}(R)>0$, then $G$ is a torsion group by Lemma 4.6, which completes the proof.

Corollary 4.8. Let $A$ be an $R$-algebra such that $A_{\text {red }} \in \mathcal{C}_{g r}^{\prime}(R)$. If $\operatorname{ch}(R)>0$, then $A \in \mathcal{C}_{g r}^{\prime}(R)$.

Lemma 4.9. Let $A=R[X] /\left(X^{2}\right)$, so that $A \in \mathcal{C}_{\mathrm{id}}^{\prime}(R)$. Then $A \in \mathcal{C}_{g r}^{\prime}(R)$ if and only if $\operatorname{ch}(R)>0$.

Proof. Suppose that $\operatorname{ch}(R)>0$. Since $A_{\text {red }}=R_{\text {red }} \in \mathcal{C}_{g r}^{\prime}(R)$, it then follows from Corollary 4.8 that $A \in \mathcal{C}_{g r}^{\prime}(R)$. Conversely, suppose that $A \in \mathcal{C}_{g r}^{\prime}(R)$, namely, $A=R[G]$ for a torsion subgroup $G$ of $U(A)$. We denote by $x$ the residue class of $X$ in $A$. Then,

$$
A=R[x]=\{a+b x \mid a, b \in R\},
$$

and hence we can write

$$
x=c_{1}\left(a_{1}+b_{1} x\right)+\cdots+c_{m}\left(a_{m}+b_{m} x\right)
$$


for some elements $a_{1}+b_{1} x, \ldots, a_{m}+b_{m} x \in G$ and $c_{1}, \ldots, c_{m} \in R$. Note that, letting $a+b x \in U(A)$ be a torsion element of order $n$, we have $(a+b x)^{n}=a^{n}+n a^{n-1} b x=1$, which implies $a^{n}=1$ and $n b=0$. Hence, there exists a positive integer $n$ such that $n b_{i}=0$ for each $i$. It then follows from (4.1) that

$$
n x=n\left(c_{1} a_{1}+\cdots+c_{m} a_{m}\right) \in R,
$$

and therefore $n=0$ in $R$. This means $\operatorname{ch}(R)>0$.

From Lemma 4.9, we know that if $\mathcal{C}_{\text {id }}^{\prime}(R) \subseteq \mathcal{C}_{g r}^{\prime}(R)$, then $\operatorname{ch}(R)>0$.

Lemma 4.10. Suppose that $\operatorname{ch}(R)>0$. Then the following conditions are equivalent:

(1) $\mathcal{C}_{\text {id }}(R) \subseteq \mathcal{C}_{g r}^{\prime}(R)$.

(2) $\mathcal{C}_{\mathrm{id}}^{\prime}(R) \subseteq \mathcal{C}_{g r}^{\prime}(R)$.

Proof. Since $\mathcal{C}_{\mathrm{id}}(R) \subseteq \mathcal{C}_{\mathrm{id}}^{\prime}(R)$, it suffices to prove $(1) \Rightarrow(2)$. If (1) holds, then for $A \in \mathcal{C}_{\text {id }}^{\prime}(R)$, we have $A_{\text {red }} \in \mathcal{C}_{\text {id }}(R)$, so that $A_{\text {red }} \in \mathcal{C}_{g r}^{\prime}(R)$. It then follows from Corollary 4.8 that $A \in \mathcal{C}_{g r}^{\prime}(R)$, and hence (2) holds.

We are thus led to seek conditions for the implication $\mathcal{C}_{\text {id }}(R) \subseteq$ $\mathcal{C}_{g r}^{\prime}(R)$. However, with a slight modification, the proof of Proposition 3.4 works for this implication; namely, we have the following:

Proposition 4.11. The following conditions are equivalent:

(1) $\mathcal{C}_{\text {id }}(R) \subseteq \mathcal{C}_{g r}^{\prime}(R)$

(2) $R^{2} \in \mathcal{C}_{g r}^{\prime}(R)$.

(3) There exist torsion elements $c_{1}, \ldots, c_{m}$ in $U(R)$ such that $R(1-$ $\left.c_{1}\right)+\cdots+R\left(1-c_{m}\right)=R$.

Corollary 4.12. Suppose that there exists a maximal ideal $M$ of $R$ such that $\operatorname{ch}(R / M)=2$ and $\mathbf{F}_{2}$ is algebraically closed in $R / M$. Then $\mathcal{C}_{\text {id }}(R) \not \subset \mathcal{C}_{g r}^{\prime}(R)$.

Proof. Let $f: R \rightarrow R / M$ be the natural surjection, and let $c$ be a torsion element in $U(R)$. Then $f(c)$ is algebraic over $\mathbf{F}_{2}$, so that 
$f(c)=1$ by assumption. Hence, $1-f(c)=0$, which implies $1-c \in M$. Therefore, $R$ does not satisfy condition (3) in Proposition 4.11.

Summarizing the results stated above, we have the following:

Proposition 4.13. The following conditions are equivalent:

(1) $\mathcal{C}_{\text {id }}^{\prime}(R) \subseteq \mathcal{C}_{g r}^{\prime}(R)$.

(2) $\operatorname{ch}(R)>0$ and $R^{2} \in \mathcal{C}_{g r}^{\prime}(R)$.

(3) $\operatorname{ch}(R)>0$, and there exist torsion elements $c_{1}, \ldots, c_{m}$ in $U(R)$ such that $R\left(1-c_{1}\right)+\cdots+R\left(1-c_{m}\right)=R$.

(4) $\operatorname{ch}(R)>0$, and there exist torsion elements $\bar{c}_{1}, \ldots, \bar{c}_{m}$ in $U\left(R_{\text {red }}\right)$ such that $R_{\text {red }}\left(1-\bar{c}_{1}\right)+\cdots+R_{\text {red }}\left(1-\bar{c}_{m}\right)=R_{\text {red }}$.

Proof. The equivalence of (1), (2) and (3) follows from Lemma 4.9, Lemma 4.10 and Proposition 4.11. The implication $(3) \Rightarrow(4)$ is clear. It thus remains to show $(4) \Rightarrow(3)$. Since the natural group homomorphism $\sigma: U(R) \rightarrow U\left(R_{\text {red }}\right)$ is surjective, there exist $c_{1}, \ldots, c_{m} \in U(R)$ such that $\sigma\left(c_{i}\right)=\bar{c}_{i}$ for each $i$. Let $J=R\left(1-c_{1}\right)+\cdots+R\left(1-c_{m}\right)$. Then $J+\operatorname{nil}(R)=R$, so that $a+b=1$ for some $a \in J$ and $b \in \operatorname{nil}(R)$. Then $(1-a)^{n}=0$ for a sufficiently large integer $n$, which implies $1 \in J$. Note that each $c_{i}$ is a torsion element of $U(R)$ by virtue of Lemma 4.6. Thus, (3) holds.

Now, combining Proposition 4.4 with Proposition 4.13, we can give conditions for $R$ to hold the equality $\mathcal{C}_{\mathrm{id}}^{\prime}(R)=\mathcal{C}_{g r}^{\prime}(R)$.

Theorem 4.14. The following conditions are equivalent:

(1) $\mathcal{C}_{\mathrm{id}}^{\prime}(R)=\mathcal{C}_{g r}^{\prime}(R)$.

(2) $\operatorname{ch}(R)>0$ and $\mathcal{C}_{g r}^{\prime}(R) \subseteq \mathcal{C}_{\mathrm{id}}^{\prime}(R)$.

(3) $\operatorname{ch}(R)>0$ and $\phi_{n}(X)$ has a root in $R_{\text {red }}$ for every positive integer $n$.

(4) $\operatorname{ch}(R)>0$ and $\phi_{p^{m}}(X)$ has a root in $R_{\text {red }}$ for every prime number $p$ and positive integer $m$.

Proof. The equivalence of (2), (3) and (4) follows from Proposition 4.4 , and the implication $(1) \Rightarrow(2)$ follows from Lemma 4.9. For (4) $\Rightarrow(1)$, it suffices to show $\mathcal{C}_{\mathrm{id}}^{\prime}(R) \subseteq \mathcal{C}_{g r}^{\prime}(R)$ because of the equivalence of (4) and (2). Set $n=\operatorname{ch}(R)$, and let $p$ be a prime number not dividing 
$n$, so that $p \in U\left(R_{\text {red }}\right)$. Then, letting $\bar{c}$ be a root of $\phi_{p}(X)$ in $R_{\text {red }}$, we know from Lemma 2.4 that $R_{\text {red }}(1-\bar{c})=R_{\text {red }}$. Thus, $\mathcal{C}_{\text {id }}^{\prime}(R) \subseteq \mathcal{C}_{g r}^{\prime}(R)$ by Proposition 4.13, which completes the proof.

We conclude this paper with some supplementary results in connection with [9].

Lemma 4.15. Suppose that $R$ is a zero-dimensional semilocal ring with maximal ideals $M_{1}, \ldots, M_{n}$. Then the following conditions are equivalent:

(1) $\mathcal{C}_{\mathrm{id}}^{\prime}(R) \subseteq \mathcal{C}_{g r}^{\prime}(R)$

(2) $\operatorname{ch}(R)>0$, and for each $i$ either (a) or (b) below holds:

(a) $\operatorname{ch}\left(R / M_{i}\right) \neq 2$;

(b) $\operatorname{ch}\left(R / M_{i}\right)=2$ and $\mathbf{F}_{2}$ is not algebraically closed in $R / M_{i}$.

(3) $\operatorname{ch}(R)>0$, and there exists a torsion element $c$ in $U(R)$ such that $1-c \in U(R)$.

Proof. Since the implications (1) $\Rightarrow(2)$ and (3) $\Rightarrow(1)$ are immediate consequences of Corollary 4.12 and Proposition 4.13, we give a proof only for $(2) \Rightarrow(3)$. Since $R$ is zero-dimensional, letting $K_{i}=R / M_{i}$, we have an $R$-algebra isomorphism

$$
f: R_{\text {red }} \longrightarrow K_{1} \times \cdots \times K_{n} .
$$

For each $i$, choose $c_{i} \in K_{i}$ as follows: If $\operatorname{ch}\left(K_{i}\right) \neq 2$, then let $c_{i}=-1$. If $\operatorname{ch}\left(K_{i}\right)=2$, then let $c_{i}$ be an element of $K_{i} \backslash \mathbf{F}_{2}$ such that $c_{i}$ is algebraic over $\mathbf{F}_{2}$; note that ord $\left(c_{i}\right)$ is finite because $\mathbf{F}_{2}\left(c_{i}\right)$ is a finite field. Let $\bar{c}$ be the element of $R_{\text {red }}$ such that

$$
f(\bar{c})=\left(c_{1}, \ldots, c_{n}\right) \in K_{1} \times \cdots \times K_{n},
$$

and let $c$ be an element of $R$ such that $\bar{c}=c \bmod \operatorname{nil}(R)$. Since ord $\left(c_{i}\right)$ is finite for each $i$, it then follows that $\bar{c}^{m}=1$ for some $m>0$, which implies $c^{m} \in 1+\operatorname{nil}(R)$. Therefore, $c$ is a torsion element of $U(R)$ by Lemma 4.6. Moreover, we have $1-c \notin M_{i}$ for any $i$, because $1-c_{i} \neq 0$ for any $i$. This means $1-c \in U(R)$, which completes the proof.

Corollary 4.16. Let $R$ be a zero-dimensional semilocal ring with maximal ideals $M_{1}, \ldots, M_{n}$, and let $A$ be an $R$-algebra that is a finite 
$R$-module. If $\operatorname{ch}(R)>0$ and $R / M_{i}$ is algebraically closed for each $i$, then $A$ is a quotient ring of $R G$ for a finite abelian group $G$.

Proof. Letting $K_{i}=R / M_{i}$ for each $i$, we have $R_{\text {red }} \cong_{R} K_{1} \times \cdots \times K_{n}$. Note that $A_{\text {red }}$ is a finite $R_{\text {red-module. Thus, we have }}$

$$
A_{\text {red }} \cong_{R} K_{1}^{r_{1}} \times \cdots \times K_{n}^{r_{n}}
$$

for some positive integers $r_{1}, \ldots, r_{n}$, so that $A_{\text {red }}=\Delta\left(A_{\text {red }}\right)$, i.e., $A \in \mathcal{C}_{\text {id }}^{\prime}(R)$. Therefore, $A$ is a quotient ring of $R G$ for a finite abelian group $G$ by Lemma 4.15 and Remark 4.2 .

\section{REFERENCES}

1. G. Higman, The units of group rings, Proc. Lond. Math. Soc. 46 (1940), 231-248.

2. I. Kaplansky, Infinite Abelian groups, University of Michigan Press, Ann Arbor, 1969.

3. G. Karpilovsky, Commutative group algebras, Dekker, New York, 1983.

4. H. Kawai, Algebras generated by idempotents and commutative group algebras over a ring, Comm. Alg. 30 (2002), 119-128.

5. Conditions for a product of residue-class rings of a ring to be generated by a p-group of units, Comm. Alg. 33 (2005), 371-379.

6. H. Kawai and N. Onoda, Commutative group algebras generated by idempotents, Toyama Math. J. 28 (2005), 41-54.

7. , Commutative group algebras whose quotient rings by nilradicals are generated by idempotents, Rocky Mountain J. Math. 41 (2011), 229-238.

8. H. Matsumura, Commutative algebra, Benjamin, New York, 1970.

9. R.D. Pollack, Over closed fields of prime characteristic, All algebras are quotients of group algebras, Expo. Math. 11 (1993), 285-287.

General Education, Ishikawa National College of Technology, Ishikawa 929-0392, JAPAN

Email address: kawai@ishikawa-nct.ac.jp

Department of Mathematics, University of Fukui, Fukui 910-8507, Japan

Email address: onoda@u-fukui.ac.jp 